GANIT J. Bangladesh Math. Soc. (ISSN 1606-3694) 36 (2016) 1-5

\title{
JORDAN DERIVATIONS ON LIE IDEALS OF $\sigma$-PRIME RINGS
}

\author{
Akhil Chandra Paul ${ }^{1}$ and Md. Mizanor Rahman ${ }^{2, *}$ \\ ${ }^{1}$ Department of Mathematics, University of Rajshahi, Rajshahi-6205, Bangladesh. \\ ${ }^{2}$ Department of Mathematics, Jagannath University, Dhaka-1100, Bangladesh. \\ *Corresponding author: mizanorrahman@gmail.com
}

Received 03.03.2015

Accepted 10.11.2015

\begin{abstract}
In this paper we prove that, if $U$ is a $\sigma$-square closed Lie ideal of a 2 -torsion free $\sigma$-prime ring $R$ and $d: R\left(R\right.$ is an additive mapping satisfying $d\left(u^{2}\right)=d(u) u+u d(u)$ for all $u \in U$ then $d(u v) \equiv d(u) v+u d(v)$ holds for all $u, v \in U$.
\end{abstract}

Keywords: Lie ideal, $\sigma$-square closed Lie ideal, $\sigma$-prime ring, Jordan derivation, derivation.

\section{Introduction}

Throughout the paper, we consider $R$ to be an associative ring with centre $Z$. $[a, b]=a b-b a$ which denotes the commutator of $a$ and $b$, we will use the identities: $[a b, c]=[a, c] b+a[b, c]$ and $[a, b c]=[a, b] c+b[a, c]$ for all $a, b, c \in R$. An additive subgroup $U$ of $R$ is called a Lie ideal if $\left[U_{i} R\right] \subseteq U_{*}$ An additive mapping $\mathbb{d}_{\mathbb{*}} R(R \quad$ is called a derivation if $d(a b)=d(a) b+a d(b)$ holds for all $a, b \in \mathbb{R}$ and it is called a Jordan derivation if $\bar{d}\left(a^{2}\right)=\bar{d}(a) a+\bar{a}(a)$ holds for all $\bar{a} \in R$. Clearly every derivation is a Jordan derivation but the converse is not true in general. A ring $R$ is said to be a prime ring if $a R b=0(a, b \in R)$ implies that $a=0$ or $b=0$. An additive mapping $f: R(R$ is called a generalized derivation with the associated derivation $d: R(R$ if $f(a b)=f(a) b+a d(b)$ holds for all $a, b \in R$; it is called a Jordan generalized derivation with the associated derivation $d$ of $R$ such that $f\left(a^{2}\right)=f(a) a+a d(a)$ holds for all $a \in R$. R. Awtar [1] proved that if $U \nsubseteq Z$ is a square closed Lie ideal of a 2-torsion free prime ring $R$ and $d: R(R$ is an additive mapping such that $d\left(u^{2}\right)=d(u) u+u d(u)$ for all $u \in V$ then $d(u v)=d(u) v+u d(v)$ holds for all $u, v \in U$.

We need the following lemmas due to R. Awtar [1] for proving our result.

Lemma 1.1 If $U \Psi Z$ is a Lie ideal of a ring $R$, then $d(u+v)=d(u) v+u d^{u}(v)+d(v) u+v u^{3}(u)$ holds for all $u, v \in U$.

Lemma 1.2 If $U \nsubseteq Z$ is a Lie ideal of a ring $R$, then $d(u u)=d(u) v u+u d(v) u+u v d(u)$ holds for all $u, v \in U$.

Lemma 1.3 If $U \nsubseteq Z$ is a Lie ideal of a ring $R$, then $d(u v w+w v u)=d(u) v w+u d(v) w+u v d(w)+d(w) v u+w d(v) u+w v d(u)$ holds for all $u_{n} v, w \in U$. 
Lemma 1.4 If $U \nsubseteq Z$ is a Lie ideal of a ring $R$, then $u^{v}[u, v]=0$ holds for all $u, v \in U$, where $u^{*}=d(u v)-d(u) v \mid u d(v)$.

Lemma 1.5 If $U \nsubseteq Z$ is a Lie ideal of a ring $R$, then $[u, v] u^{v}=0$ for all $u, v \in U$, where $u^{v}$ is as in Lemma1.4

\section{Jordan Derivations on Lie Ideals of $\sigma$-Prime Rings}

Let $R$ be a ring. A mapping $(: R(R$ is called an involution if $((a+b)=((a)+((b),(2(a)=a$ and $\left((a b)=((b))\left((a)\right.\right.$ holds for all $a_{z} b \in R$. A Lie ideal $U$ of $R$ is called a $\sigma$-Lie ideal if $((U)=U$ and it is called a $\sigma$-square closed Lie ideal if it is a $\sigma$-Lie ideal and for all $u \in U, u^{2} \in U$. A ring $R$ with involution $\sigma$ is said to be a $\sigma$-prime ring if $a R b=a R((b)=\{0\}$ implies that $a=0$ or $b=0$. It is worthwhile to note that every prime ring having an involution $\sigma$ is $\sigma$ prime but the converse is not true in general. As an example, let $T=R \times R^{0}$, where $R^{0}$ is an opposite ring of a prime ring $R$ with involution $(x, y)=(y, x)$. Then $\mathrm{T}$ is not prime if $(0, a) T(a, 0)=0$. But, $R$ is $\sigma$-prime if we set $(a, b) T(x, y)=0$ and $(a, b) T(((x, y))=0$, then $a R x \times y R b=0$ and $a R \bar{R} y \times x R b=0$ and thus $a R x=y R b=a R y=x R b=0$ by Oukhtite and Salhi [6]. We define the set $S_{1} a \sigma(R)=\{x(R:((x)= \pm x\}$ which are known as the set of symmetric and skew symmetric elements of $R$. Let $U$ be a Lie ideal of $R$. We define $C_{R}(U)=\{r \in R: m=u r, \forall u \in U\}$ which we shall call the centralizer of $U$ with respect to $R$. Oukhtite and Salhi [12] worked on left derivation on $\sigma$-prime rings and proved that $U \subseteq Z$ or $d(U)=\mathbf{0}$, where $U$ is a nonzero $\sigma$-square closed Lie ideal of $R$. Oukhtite and Salhi [12] described additive mappings $d: R\left(R\right.$ such that $d^{2}\left(u^{2}\right)=2 u d^{\prime}(u) \vee u \in U$, where $U$ is a nonzero $\sigma$-square closed Lie ideal of a 2 -torsion free $\sigma$-prime ring $R$ and prove that $d(u v)=u d(v)+v d(u)$ for all $u, v \in U$. Afterwords, Oukhtite, Salhi and Taoufiq [11] studied Jordan generalized derivations on $\sigma$-prime rings and proved that every Jordan generalized derivation on $U$ of $R$ is a generalized derivation on $U$ of $R$, where $U$ is a $\sigma$-square closed Lie ideal of a 2-torsion free $\sigma$-prime ring $R$. Some significant results on Lie ideals and generalized derivations in $\sigma$-prime rings have been obtained by M. S. Khan and M. A. Khan [5]. On the other hand, various remarkable characterizations of $\sigma$-prime rings on $\sigma$-square closed Lie ideals have been studied by many authors viz. M. R. Khan, D. Arora and M. A. Khan [4] ; Oukhtite and Salhi [7, 8, 9,10] and J. Bergun, I. N. Herstein and J. W. Kerr [2] and I. N. Herstein [3]. In this paper, we shall prove that if $d: R \rightarrow R$ is an additive mapping satisfying $d\left(u^{2}\right)=\mathbf{2} u d(u) \mathbf{v} u \in U$, where $U$ is a $\sigma$-square closed Lie ideal of a 2-torsion free $\sigma$-prime ring $R$ then $d(u v)=u d(v)+v d(u)$ for all $u_{w} v \in U$ and hence every Jordan derivations on a $\sigma$-prime ring $R$ is a derivation on $R$. We begin with the following results.

Lemma 2.1 Let $R$ be a 2-torsion free $\sigma$-prime ring and $U$ be a $\sigma$-Lie ideal of $R$. Let $u \in U$ be any element such that $[u,[u, x]]=0$, for all $x \in R$, then $[u, x]=0$.

Proof: We have $[u[u, x]]=\mathbf{0}$ for all $x \in R$. Let $y \in R$, then $x y \in R$. Replacing $x$ by $x y$, we have $[u,[u, x y]]=0$. So $0=[u,[u, x y]]=[u, x[u, y]+[u, x] y]$ 


\section{$=[u, x[u, y]]+[u,[u, x] y]$ \\ $=x[u[u, y]]+[u, x][u, y]+[u, x][u, y]+[u,[u, x]] y$ \\ $=2[u, x][u, y]$.}

Since $R$ is 2-torsion free so $[u, x][u, \quad y]=0$. For every $z \in R$ we have $z y \in R$. Putting $z x$ for $y$, we have $[u, x][u, \quad z x]=0$. Therefore, $0=[u, x](z[u, x]+[u, z] x)$

$$
\begin{aligned}
& =[u, x] z[u, x]+[u, x][u, z] x \\
& =[u, x] z[u, x] .
\end{aligned}
$$

Therefore, $[u, x] R[u, x]=0$. Since $((U)=U$, we have $((u)=u$, for all $u \in U$. Let $x \in S_{a \sigma}(R)$. Then $((x)= \pm x$. If $((u)=u$ and $((x)=-x$, then

Hence $[u, x] R[u, x]=[u, x] R([u, x]=0$. By the $\sigma$-primeness of $R$, we get $[u, x]=0$,

Lemma 2.2 Let $R$ be a 2-torsion free $\sigma$-prime ring and $U \neq 0$ be a $\sigma$-Lie ideal and a $\sigma$-subring of $R$. Then either $U \subseteq Z$ or $U$ contains a nonzero $\sigma$-ideal of $R$.

Proof: First we assume that, $U$ as a $\sigma$-ring is not commutative. Then for some $u, v \in U,[u, v] \neq 0$ and $[u, v] \in U$. Therefore the ideal $J$ of $R$ generated by $[u, v]$ is nonzero, $J \subseteq U$ and $(O D=J$. On the other hand, let us assume that $U$ is commutative. Then for every $u \in U,[u,[u, x]]=\mathbf{0}$ for all $x \in R$. Hence by Lemma 2.1, $[u, x]=0$. This shows that $U \subseteq Z$.

Lemma 2.3 If $U \nsubseteq Z$ is a $\sigma$-Lie ideal of a $\sigma$-prime ring $R$, then $C_{R}(U)=Z$.

Proof: $C_{\bar{R}}(U)$ is both a $\sigma$-subring and a $\sigma$-Lie ideal of $R$ and $C_{\bar{R}}(U)$ contains no nonzero $\sigma$-ideal of $R$. In view of Lemma 2.2, $C_{R}(U) \subseteq Z$. Therefore, $C_{R}(U)=Z$.

Lemma 2.4 If $U$ is a $\sigma$-Lie ideal of a $\sigma$-prime ring $R$ and $a \in R$. If $[a,[U, U]]=\mathbf{0}$ then $[a, U]=0$, that is, $C_{R}([U, U])=C_{R}(U)$.

Proof: If $[U, U] \Phi Z$, then by Lemma 2.3, $a \in Z$, so a centralizes $U$. On the other hand, let $[U, U] \subseteq Z_{z}$ then we have $[u,[u, x]]=\mathbf{0}$ for $u \in U$ and $x \in R$. In view of Lemma 2.1, $[u, x]=0$. This yields that $U \subseteq Z$. For both the cases we have seen that $a \in C_{R}(U)$. This gives that $C_{\bar{R}}([U, U])=C_{R}(U)$.

Lemma 2.5 Let $U \Phi Z$ be a $\sigma$-square closed Lie ideal of a 2 -torsion free $\sigma$-prime ring $R$ and $d: R\left(R\right.$ be an additive mapping satisfying $d\left(u^{2}\right)=d(u) u+u d(u)$, for all $u \in U$. If $u^{v} \equiv d(u v) d(u) v-u d(v)$, for all $u_{*} v \in U$ then $u^{v} w[u v]=0$, for all $w \in U$.

Proof: In view of Lemmas 1.4 and 1.5, we have $\left[u^{v}, \quad[u, v]\right]=u^{v}[u, v]-[u, v] u^{v}=0$. This yields that $u^{v} \in C_{R}([U, U])=C_{R}(U)$, by Lemma 2.4. Hence for every $w \in U$, we have $u^{v} w[u, v]=0$. 
Lemma 2.6 ([7], Lemma 2.2) Let $U \nsubseteq Z$ be a $\sigma$-Lie ideal of a 2-torsion free $\sigma$-prime ring $R$ and $a, b \in \bar{R}$ such that $a U b=a U((b)=0$, then $a \equiv 0$ or $b=0$.

Theorem 2.7 Let $U$ be a $\sigma$-square closed Lie ideal of a 2-torsion free $\sigma$-prime ring $R$ and $d: R\left(R\right.$ be an additive mapping satisfying $d\left(u^{2}\right)=d(u) u+u d^{2}(u)$, for all $u \in U$, then $d(u v)=d(u v+u d(v)$ holds for all $u, v \in U$.

Proof: If $U$ is a non-commutative Lie ideal of $R$ then $U \nsubseteq Z$. By Lemma 2.5, we have $a^{b} w[a, b]=0$ for all $a, b, w \in U$. Let us assume that $a, b \in U \cap S_{a \sigma}(R)$. Since $((U)=(U)$, we have $([a, b]=[a, b] \quad$ as $[a, b] \in U$. If $((b)=\mathbf{I} b$ and $((a)=a$, then Also, if $\left((b)=b \quad\right.$ and $\quad\left((a)=\mid a_{k}\right.$ then $\left([a, b]=[a, b]\right.$. Therefore, we have $a^{\mathbb{T}} b w[a, b]=a^{\top} b w([a, b]=0$. By applying the Lemma 2.6 in the above relation, we obtain that $a^{b}=\mathbf{0}$ or $[a, b]=0$ for all $a, b \in U \cap S_{a \sigma}(R)$. Let

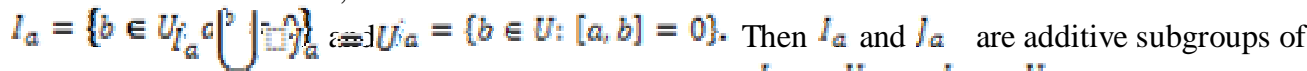
U such that . Then by Brauer's trick $t_{a}=U$ or $l_{a}=U$. Using the similar argument, we have $U=\left\{a \in U: U=I_{a}\right\}$ or $U=\left\{a \in U: U=J_{a}\right\}$. If $U=\left\{a \in U: U=J_{a}\right\}$ then $[a, b] \equiv 0$, which yields that $U \subseteq Z$, by Lemma 2.2. Which is a contradiction to the fact that $U \nsubseteq Z$. So we have $U=\left\{a \in U: U=I_{a}\right\}$ and hence $a^{b}=0$ for all $a, b \in U \cap S_{a \sigma}(R)$. This implies

$$
d(a b)=d(a) b+a d(b), \forall a, b\left(U \cap S_{1} a \sigma(R) \ldots \ldots\right.
$$

$$
\begin{aligned}
& \text { Now let } u, v \in U \text {. If we define } \\
& u_{1} 1=u+\left((u), \quad u_{1} 2=u \mathbf{I}\left((u), v_{1} 1=v+\left((v), v_{1} 2=v \mathbf{I}((v) . \quad \text { Then }\right.\right.\right. \\
& u_{1}, u_{2}, v_{1}, v_{2} \in U \cap S_{a g}(R) \text { and we have } 2 u=u_{1}+u_{2}, 2 v=v_{1}+v_{2}+\text { Therefore, in }
\end{aligned}
$$
view of (1), we obtain

$$
\begin{aligned}
& d(2 u 2 v)=d\left(u_{1} v_{1}+u_{1} v_{2}+u_{2} v_{1}+u_{2} v_{2}\right) \\
& =d\left(u_{1}\right) v_{1}+u_{1} d\left(v_{1}\right)+d\left(u_{1}\right) v_{2}+u_{1} d\left(v_{2}\right)+d\left(u_{2}\right) v_{1}+u_{2} d\left(v_{1}\right)+d\left(u_{2}\right) v_{2}+u_{2} d\left(v_{2}\right) \\
& =\left(d\left(u_{1}\right)+d\left(u_{2}\right)\right) v_{1}+\left(u_{1}+u_{2}\right) d\left(v_{1}\right)+\left(d\left(u_{1}\right)+d\left(u_{2}\right)\right) v_{2}+\left(u_{1}+u_{2}\right) d\left(v_{2}\right) \\
& \quad=d\left(u_{1}+u_{2}\right) v_{1}+2 u d\left(v_{1}\right)+d\left(u_{1}+u_{2}\right) v_{2}+2 u d\left(v_{2}\right)=d(2 u) v_{1}+2 u d\left(v_{1}\right)+d(2 u) v_{2}+2 u d\left(v_{2}\right)=2 d \\
& =2 d(u) 2 v+2 u d(2 v) \\
& =\mathbf{4} d(u) v+4 u d(v) . \\
& \text { Thus } 4 d(u v)=4(d(u) v+u d(v)) \text {. Since } R \text { is 2-torsion free, we obtain }
\end{aligned}
$$

$d(w)=d(u) v+u d(v) . \quad$ If $U$ is a commutative $\sigma$-Lie ideal of $R$, then by Lemma 2.2,

$U \subseteq Z$. Therefore, by using 2-torsion freeness of $R$ and in view of the Lemma 1.1, we have

$d(u v)=d(u) v+u d(v)$. 
In view of above theorem, we obtain the following corollary.

Corollary 2.8 Let $R$ be a 2 -torsion free $\sigma$-prime ring. Then every Jordan derivations on $R$ is a derivation on $R$.

\section{REFERENCES}

[1] Awtar, R., Lie ideals and Jordan derivations of prime rings, Proc. Math. Soc. 90(1) (1984), 9-14.

[2] Bergun, J., Herstein, I. N. and Kerr, J. W., Lie ideals and derivations of prime rings, J. Algebra, 71 (1981), 259-267.

[3] Herstein, I. N., Topics in Ring Theory, The University of Chicago Press, Chicago, 111 -London, 1969.

[4] Khan, M. R., Arora, D. and Khan, M. A., Notes on derivations and Lie ideals in sigma-prime rings, Advances in Algebra, 3(1) (2010), 19-23.

[5] Khan, M. S. and Khan, M. A., Lie ideal and generalized derivations in sigma-prime rings, J. Algebra, 6(29) (2012), 1419- 1429.

[6] Oukhtite, L. and Salhi, S., On generalized derivations of sigma-prime rings, African Diaspora J. Math., 5(1) (2006), 19-23.

[7] Oukhtite, L. and Salhi, S., On commutativity of sigma-prime rings, Glasnik Mathematicki, 41(1) (2006), 57-64.

[8] Oukhtite, L and Salhi, S., Derivations and commutativity of sigma-prime rings, Int. J. Contemp., 1(2006), 439-448.

[9] Oukhtite, L. and Salhi, S., On derivations in sigma-prime rings, Int. J. Algebra, 1(2007), 241-246.

[10] Oukhtite, L. and Salhi, S., Sigma-Lie ideals with derivations as homomorphisms and antihomomorphisms, Int. J. Algebra, 1(2007), 235-239.

[11] Oukhtite, L., Salhi, S. and Taofiq, L., Jordan generalized derivations on sigma-prime rings, Int. J. Algebra, 1(5) (2007), 231-234.

[12] Oukhtite, L. and Salhi, S., Centralizing automorphisms and Jordan left derivations on sigma-prime rings, Advances in Algebra, 1(1) (2008), 19-26. 